\title{
Ultrasound Cycloplasty in Chinese Glaucoma Patients: Results of a 6-Month Prospective Clinical Study
}

\author{
Qin Luo ${ }^{a}$ Wenwen Xue ${ }^{a}$ Yulan Wang ${ }^{a}$ Bin Chen ${ }^{a}$ Shuangshuang Wang ${ }^{b}$ \\ Yiping Dong ${ }^{\mathrm{a}}$ Yan $\mathrm{Ru}^{\mathrm{a}}$ Ling Ge ${ }^{\mathrm{a}}$ \\ aDepartment of Ophthalmology, Shanghai Eye Disease Prevention \& Treatment Center, Shanghai, China; \\ bShanghai Pharmacies Medical \& Biotechnology Co., Ltd, Shanghai, China
}

\section{Keywords}

Ultrasound cycloplasty · Glaucoma · Intraocular pressure .

Ciliary body $\cdot$ Noninvasive procedure

\begin{abstract}
Purpose: The aim of this study was to evaluate the shortterm efficacy and safety of ultrasound cycloplasty (UCP) procedure in Chinese glaucoma patients. Methods: As a singlecenter, prospective, noncomparative study, 23 eyes of 23 patients suffering from glaucoma with uncontrolled intraocular pressure (IOP) $\geq 21 \mathrm{~mm} \mathrm{Hg}$ underwent a multidose UCP treatment with the activations of 6,8 , or10 sectors. Types of glaucoma include primary open-angle glaucoma (7/23), primary angle closure glaucoma (PACG) $(9 / 23)$, and secondary glaucoma (SG) (7/23). A complete ophthalmic examination including intraocular pressure (IOP) measurements was performed before UCP procedure and at 1 day, 1 month, 3 months, and 6 months after the procedure. An IOP reduction of $\geq 20 \%$ and IOP $>5 \mathrm{~mm} \mathrm{Hg}$ without increasing hypotensive medication at the follow-up visit was defined as therapeutic success. The postoperative complications were also recorded and compared to baseline for safety
\end{abstract}

Karger@karger.com www.karger.com/ore

Karger ${ }^{\prime \prime} \div$

GOPEN ACCESS
(C) 2021 The Author(s)

Published by S. Karger AG, Basel

This is an Open Access article licensed under the Creative Commons Attribution-NonCommercial-4.0 International License (CC BY-NC) (http://www.karger.com/Services/OpenAccessLicense), applicable to the online version of the article only. Usage and distribution for commercial purposes requires written permission. evaluation. Results: The mean baseline IOP of 23 treated eyes was $37.2 \pm 12.1 \mathrm{~mm} \mathrm{Hg}$. The IOP reduction after UCP procedure was $23,49,33$, and $34 \%$ at 1 day, 1 month, 3 months, and 6 months, respectively. Thus, the corresponding overall therapeutic success rates reached $61 \%(14 / 23)$, $83 \%(19 / 23), 65 \%(15 / 23)$, and $61 \%(14 / 23)$, respectively. Baseline IOPs of 8- and 10-sector groups $(37.0 \pm 9.9 \mathrm{~mm} \mathrm{Hg}$ and $50.1 \pm 12.2 \mathrm{~mm} \mathrm{Hg}$ ) were significantly higher than that of 6 sector groups ( $30.1 \pm 8.2 \mathrm{~mm} \mathrm{Hg}$ ). Therapeutic success rates of $6-, 8-$, and 10 -sector groups reached $44 \%$ (4/9), $56 \%$ $(5 / 9)$, and $100 \%(5 / 5)$, respectively. There were the highest percentage of IOP reduction (50 and $41 \%$ ) and therapeutic success rate $(6 / 7 ; 86 \%$ and $7 / 9 ; 78 \%)$ in the SG group and PACG group, respectively. In addition, preoperative ocular pain symptoms of 4 patients were all disappeared within 1 week after UCP. No serious intraoperative or postoperative complications occurred. Conclusion: UCP procedure is an effective and well-tolerated treatment to reduce IOP in Chinese glaucoma patients, which offered a novel alternative for glaucoma treatment.

(c) 2021 The Author(s).

Published by S. Karger AG, Basel

Qin Luo and Wenwen Xue contributed equally to the manuscript.
Correspondence to:

Ling Ge, geling99@sina.com 


\section{Introduction}

As an irreversible progressive optic neuropathy, glaucoma affects more than 70 million people worldwide and becomes the second leading cause of blindness in the world [1]. Due to the positive correlation between glaucoma prevalence and advanced age, glaucoma will become a heavy public health burden in the coming decades, especially in China with the largest aging population [2]. Glaucoma is characterized by progressive structural and functional abnormalities of the optic nerve and typical appearance patterns of visual field damage if untreated. Elevated intraocular pressure (IOP) is considered to be the most critical risk factor for glaucoma, and IOP reduction is the only proven treatment to be effective to prevent vision impairment [3].

IOP reduction can be achieved by topical or systemic medications, laser therapies, and incisional surgeries, which aim to balance the inflow and outflow of aqueous humor circulation by decreasing production or facilitating drainage [4]. Although glaucoma treatment usually begins with hypotensive medications, in order to prevent visual field defect from progressing as soon as possible, surgical treatment often regarded as the first-line therapy for many patients with severe glaucoma, but some patients may refuse incisional surgery because of different kind of reasons. Cyclodestructive therapeutic methods for glaucoma aim to partially destroy the ciliary body and induce the coagulation necrosis of secretory ciliary epithelium, following heating (laser and microwave) or freezing (cryotherapy) [5]. Most current physical methods have 2 technical application restrictions including nonselectivity for the target tissue and unpredictability for the dose-effect relationship, and they may be associated with serious complications, including chronic hypotony, vision loss, uveitis, and more rarely phthisis bulbi [6]. Recently introduced in China, a new noninvasive glaucoma procedure called ultrasound cycloplasty (UCP) using high-intensity focused ultrasound has been previously proven to be a selective and precise method of ciliary body coagulation leading to effective IOP reduction with well tolerance and causing less damage to adjacent tissues while treating nonoptically transparent structures [7]. UCP decreases IOP by reducing aqueous humor production and by increasing uveoscleral outflow. Many published studies have reported positive and favorable results of UCP procedure regarding safety and efficacy in patients with refractory glaucoma or patients who were not surgically treated before [7-18]. In the present study, the objective was to evaluate, in Chinese patients with medically uncontrolled glaucoma with low or good vision, the efficacy and safety of UCP treatment and to explore the feasibility of multidose UCP.

\section{Materials and Methods}

\section{Ethical Approval}

According to the principles of the declaration of Helsinki and the World Glaucoma Association guidelines, this single-center pilot clinical study was conducted from June 2018 to November 2019 , and all participants were provided with written informed consent before any study procedure.

\section{Patient Enrollment}

Patients who were selected to undergo the procedure were all diagnosed with primary or secondary, open angle or angle closure glaucoma. Inclusion criteria were as follows: age greater than 18 years old with primary or secondary glaucoma; baseline IOP $\geq 21$ $\mathrm{mm} \mathrm{Hg}$ under medical treatment, with or without previous filtering surgery; and ability and willingness to complete all postoperative follow-up visits. Exclusion criteria were as follows: ocular infection in 2 weeks before UCP procedure; other disease that can affect IOP evaluation; pregnancy; and serious systemic disease.

\section{Baseline Evaluation}

Baseline examination included visual acuity, noncontact tonometry with 3 measurements, slit-lamp biomicroscopy with gonioscopy, and fundus examination. Preoperative investigations included white-to-white parameters of the corneal diameter and axial length measurements, pachymetry (IOL Master ${ }^{\circledR} 700$ Carl Zeiss Meditech), and ultrasound biomicroscopy. In addition, patient demographic information, glaucoma symptoms, and best corrected visual acuity (BCVA) were recorded.

\section{UCP Procedure}

As previous studies described [6, 7], UCP procedures were performed by the same surgeon, under retrobulbar anesthesia, using EyeOP $1{ }^{\circledR}$ device (Eye Tech Care, France). The single used device is composed of 2 parts: (1) a coupling cone which is positioned and centered on the ocular surface and stabilized during all the procedure by a low-level vacuum ring, and (2) a therapy probe equipped with 6 piezoelectric transducers, inserted in the upper part of the cone. EyeOP1 is available in 3 diameters $(11,12$, and $13 \mathrm{~mm}$ ), selected according to ocular parameters (axial length and white-towhite measurements obtained by optical biometry (IOL Master ${ }^{\circledR}$ Carl Zeiss Meditech) and ultrasound biomicroscopy. After filling the device with saline solution, the procedure was initiated by pressing on the foot pedal and the transducers were activated sequentially. The following standard parameters of operation module were applied: operating frequency, $21 \mathrm{MHz}$; acoustic power, $2.45 \mathrm{~W}$; ultrasound exposure time per sector, $8 \mathrm{~s}$; and sequential sector activation interval, $20 \mathrm{~s}$. There were 3 modes including 6 sectors, 8 sectors, and 10 sectors activated being applied for UCP treatment according to the preoperative IOP and vision status. After the first activation of the 6 transducers successively, the 8- or 10 -sector modes were achieved by rotating the probe to the second position, and then 2 or 4 of the 6 transducers were activated again to complete the procedure $[9,10]$. 
Table 1. Patient characteristics

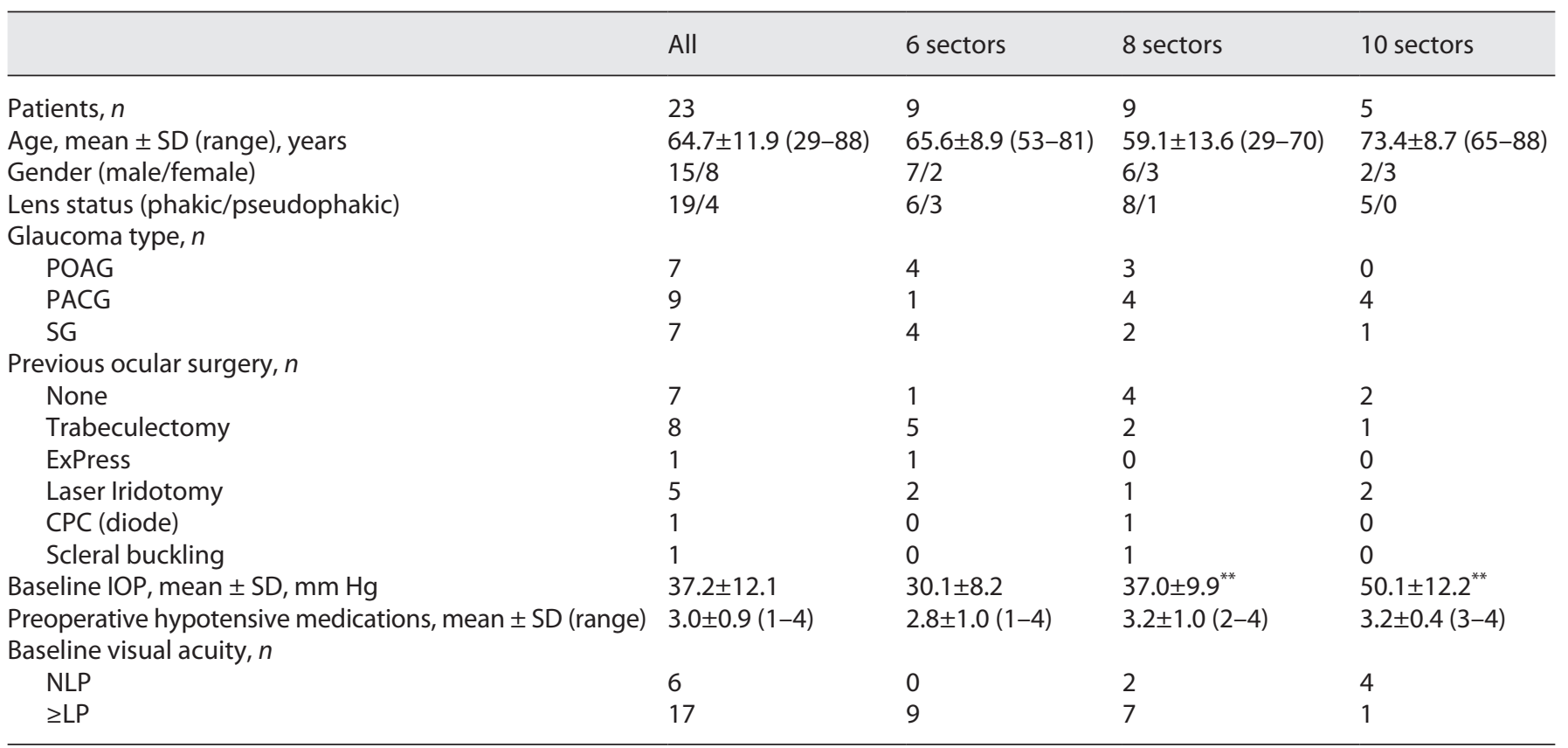

POAG, primary open-angle glaucoma; PACG, primary angle closure glaucoma; SG, secondary glaucoma; IOL, intraocular lens; IOP, intraocular pressure; NLP, no light perception; LP, light perception; SD, standard deviation. ${ }^{* *} p<0.01$ versus 6 -sector group.

Postoperatively, patients were treated with anti-inflammatory eyedrops (TobraDex, tobramycin-dexamethasone suspension) 4 times a day for 1 month. If necessary, low concentration steroid eyedrops $(0.1 \%$ fluorometholone ophthalmic suspension) were prescribed for another 1 month. No patients were prescribed with cycloplegics or pain killer after UCP treatment.

\section{Follow-Up}

Postoperative follow-up visits lasted for 6 months, which were scheduled at 1 day, 1 week, 1 month, 3, and 6 months after UCP procedure. According to the baseline examination data, IOP measurements, complications monitoring, and medication adjustment were all recorded at each visit. All postoperative IOPs were measured at the same time of day (8-10 a.m.) as the baseline IOP examination.

\section{Outcome Evaluation}

The primary outcome was mainly based on IOP reduction of the last follow-up visit. Therapeutic success was defined as an IOP reduction from baseline by a minimum of $20 \%$ compared to baseline and final IOP greater than $5 \mathrm{~mm} \mathrm{Hg}$ without adding hypotensive medications or re-intervention. Therapeutic failure was defined as an IOP reduction less than $20 \%$ compared to baseline. Secondary outcomes were based on postoperative glaucoma medication requested, visual acuity, and complications profile. The occurrence of postoperative complications, such as conjunctival hyperemia, scleral marks, ocular pain, and corneal edema were recorded and analyzed.

Snellen BCVA data were converted to logarithm of the minimum angle of resolution units (logMAR). Values of 2.0, 2.4, 3.0, and 3.5 were assigned to visual acuities of counting fingers, hand movements, light perception (LP), and no light perception (NLP), respectively.

\section{Statistical Analysis}

Quantitative data, expressed as mean \pm standard deviation, were analyzed by Student's $t$-test and $\chi^{2}$ test using statistical software (SPSS version 17.0, USA). Statistical significance was set to $p$ $<0.05$.

\section{Results}

\section{Patient Characteristics}

The patients' characteristics are listed in Table 1. Twenty-three patients were enrolled and treated with UCP treatment (9/9/5 in 6-/8-/10-sector groups), and only one eye of each patient underwent UCP procedure. The mean age and preoperative IOP of all patients were $64.7 \pm 11.9$ years old and $37.2 \pm 12.1 \mathrm{~mm} \mathrm{Hg}$. Types of glaucoma include primary open-angle glaucoma $(7 / 23)$, primary angle closure glaucoma (PACG) (9/23), and secondary glaucoma (SG) (7/23). There were 8 patients who had no surgical history for glaucoma before UCP procedure. The preoperative visual acuity of 6 patients was NLP. All patients were evaluated at month 6 , and none of 
Table 2. Baseline and postoperative mean IOP and hypotensive glaucoma medications in all and responsive patients

\begin{tabular}{|c|c|c|c|c|c|c|c|c|c|}
\hline & $\begin{array}{l}\text { mean IOP, } \\
\mathrm{mm} \mathrm{Hg}\end{array}$ & $\begin{array}{l}\text { relative IOP } \\
\text { reduction (\%) }\end{array}$ & $\begin{array}{l}\text { glaucoma } \\
\text { medications, } \\
n\end{array}$ & $\begin{array}{l}\text { success } \\
\text { rate (\%) }\end{array}$ & $p$ value ${ }^{* *}$ & $\begin{array}{l}\text { mean IOP, } \\
\mathrm{mm} \mathrm{Hg}\end{array}$ & $\begin{array}{l}\text { relative IOP } \\
\text { reduction (\%) }\end{array}$ & $\begin{array}{l}\text { glaucoma } \\
\text { medications, } \\
n\end{array}$ & $\begin{array}{l}p \\
\text { value** }\end{array}$ \\
\hline Baseline & $37.2 \pm 12.1$ & & 3.0 & & & $43.7 \pm 10.3$ & & 3.5 & \\
\hline D1 & $26.7 \pm 13.2$ & 23 & 2.9 & 61 & 0.0056 & $30.0 \pm 15.1$ & 27 & 3.2 & 0.0017 \\
\hline M6 & $22.0 \pm 7.2$ & 34 & 2.7 & 61 & $<0.001$ & $20.4 \pm 7.5$ & 51 & 2.8 & 0.001 \\
\hline
\end{tabular}

IOP, intraocular pressure. ${ }^{* *}$ Wilcoxon test IOP versus baseline. ${ }^{* *}$ Responsive patients $=$ therapeutic success.

Table 3. Baseline and postoperative mean IOP in patients at 6 months after UCP procedure according to number of sectors, types of glaucoma, and surgical glaucoma history

\begin{tabular}{llllll}
\hline & $\begin{array}{l}\text { Patients, } \\
n\end{array}$ & $\begin{array}{l}\text { Baseline IOP } \\
\text { in mm Hg } \\
\text { (mean No. } \\
\text { antiglaucoma } \\
\text { medications) }\end{array}$ & $\begin{array}{l}\text { IOP at 6 months } \\
\text { in mm Hg } \\
\text { (mean No. antiglaucoma } \\
\text { medications) }\end{array}$ & $\begin{array}{l}\text { IOP reduction } \\
\text { at } 6 \text { months, \% }\end{array}$ & $\begin{array}{l}\text { Success } \\
\text { rate, } \%\end{array}$ \\
\hline 6 sectors & 9 & $30.1 \pm 8.2(2.8)$ & $20.4 \pm 3.2(3.0)$ & 27 & 44 \\
8 sectors & 9 & $37.0 \pm 9.9(3.2)$ & $23.5 \pm 8.5(2.9)$ & 30 & 56 \\
10 sectors & 5 & $50.1 \pm 12.2(3.2)$ & $22.1 \pm 10.6(2.0)$ & 55 & 100 \\
POAG & 7 & $25.6 \pm 6.2(2.6)$ & $22.8 \pm 4.7(2.9)$ & 10 & 78 \\
PACG & 9 & $43.3 \pm 13.6(3.1)$ & $22.8 \pm 10.7(2.1)$ & 41 & 86 \\
SG & 7 & $40.8 \pm 4.9(3.4)$ & $20.2 \pm 3.7(3.4)$ & 50 & 67 \\
Surgical history & 15 & $34.9 \pm 10.9(3.0)$ & $19.6 \pm 6.0(2.7)$ & 36 & 50 \\
None surgical history & 8 & $41.4 \pm 13.8(3.1)$ & $26.5 \pm 7.5(2.9)$ & 30 & 78 \\
\hline
\end{tabular}

POAG, primary open-angle glaucoma; PACG, primary angle closure glaucoma; SG, secondary glaucoma; IOP, intraocular pressure; UCP, ultrasound cycloplasty.

them was submitted to an additional glaucoma surgical procedure or cyclodestructive procedure during the follow-up period.

\section{Efficacy}

As shown in Table 2, a significant mean IOP reduction of $23 \%$ was observed at the first day after UCP procedure. Moreover, the mean IOP significantly reduced from 37.2 $\pm 12.1 \mathrm{~mm} \mathrm{Hg}$ at baseline to $23.1 \pm 10.6 \mathrm{~mm} \mathrm{Hg}$ and 22.0 $\pm 7.2 \mathrm{~mm} \mathrm{Hg}$ at 3 and 6 months after UCP treatment, thus the therapeutic success rates reached $65 \%$ ( 15 of 23 eyes) and $61 \%$ (14 of 23 eyes), respectively. In responsive patients at month 6 , the mean IOP was significantly decreased from $43.7 \pm 10.3 \mathrm{~mm} \mathrm{Hg}$ to $21.3 \pm 8.1 \mathrm{~mm} \mathrm{Hg}$ and $20.4 \pm 7.5 \mathrm{~mm} \mathrm{Hg}$ at 3 and 6 months, corresponding to the relative IOP reductions of 49 and 51\%. From the de- creasing trend of postoperative IOP, the most significant IOP reduction of $62 \%$ was at 1 month after UCP treatment. In addition, the mean number of postoperative hypotensive medications was decreased from 3.0 to 2.7 in the overall population and 3.5 to 2.8 in the therapeutic success patients.

According to number of sectors, types of glaucoma, and surgical glaucoma history, the comparison between baseline and postoperative 6-month mean IOP are summarized in Table 3 and Figure 1. Baseline IOP of 10-sector group $(50.1 \pm 12.2 \mathrm{~mm} \mathrm{Hg})$ was significantly higher than that of the 8-sector group $(37.0 \pm 9.9 \mathrm{~mm} \mathrm{Hg})(p<0.01)$, which was higher than that of the 6-sector group (30.1 \pm $8.2 \mathrm{~mm} \mathrm{Hg})(p<0.01)$. The percentages of IOP reduction were 27,30 , and $55 \%$ in $6-, 8-$, and 10 -sector groups, respectively. In terms of therapeutic success rate of UCP 
Table 4. Preoperative and postoperative complications

\begin{tabular}{llll}
\hline Complication & Preoperative, $n$ & $\begin{array}{l}\text { 1-day } \\
\text { postoperative, } n\end{array}$ & $\begin{array}{l}\text { 6-month } \\
\text { postoperative, } n\end{array}$ \\
\hline Conjunctival hyperemia & 0 & 23 & 1 \\
Scleral marks & 0 & 1 & 0 \\
Corneal edema & 0 & 2 & 0 \\
Ocular pain & 4 & 5 & 0 \\
Induced astigmatism (>1.5 D) & 0 & 0 & 1 \\
Hypotony* & 0 & 0 & 0 \\
Choroidal detachment & 0 & 0 & 0 \\
\hline
\end{tabular}

* Hypotony $=\mathrm{IOP} \leq 5 \mathrm{~mm} \mathrm{Hg}$.

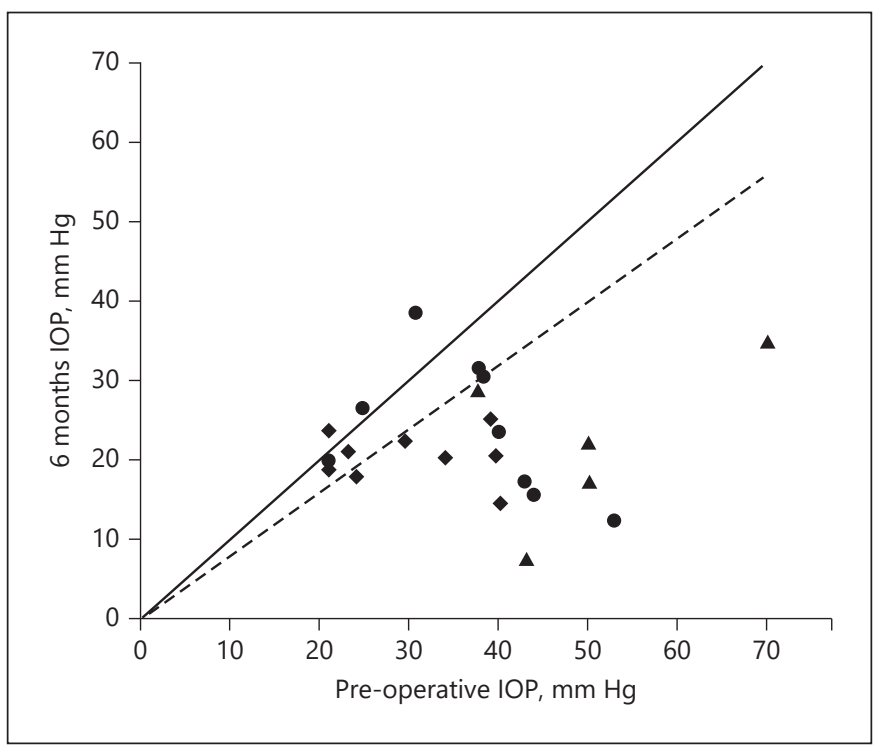

Fig. 1. Scattergram of IOP ( $\mathrm{mm} \mathrm{Hg}$ ) before UCP versus 6 months for the overall population (UCP procedure: $\triangleleft$ low dose, $\rightarrow$ medium dose, and $\boldsymbol{\Delta}$ high dose). All points below the dashed line represent eyes with IOP reduction higher than $20 \%$ compared to baseline value. IOP, intraocular pressure; UCP, ultrasound cycloplasty.

treatment, the 10 sector groups reached $100 \%$ ( 5 of 5 eyes) which were significantly higher than $56 \%$ ( 5 of 9 eyes and 4 of 9 eyes) in 8 - and 6-sector groups $(p<0.01)$. Moreover, there were the highest percentage of IOP reduction (50 and $41 \%)$ and therapeutic success rate $(6 / 7 ; 86 \%$ and $7 / 9$; $78 \%)$ in the SG group and PACG group, respectively. In addition, there was no significant difference between patients with or without glaucoma surgical history in percentage of IOP reduction ( $36 \%$ or $30 \%)$ and therapeutic success rate $(67 \%, 10$ of 15 eyes or $50 \%, 4$ of 8 eyes).

In the overall population, the number of anti-glaucoma medications was not significantly reduced at month 6 compared to the preoperative number of medications.
The number of antiglaucoma medications was significantly lower after UCP in the subgroup of patients treated with 10 sectors at 6 months than the preoperative number (mean $=3.2$ preoperatively vs. 2.0 at month 6 ; $p<0.01)$.

\section{Safety}

As shown in Table 4, the preoperative and postoperative complications including at 1 day and 6 months after UCP treatment were summarized. There were 4 glaucoma patients with preoperative ocular pain, which was relieved significantly accompanied by a significant IOP reduction at 1 day after UCP treatment. The remaining patients with ocular pain were relieved spontaneously within 1 week. As the common and minor complication in glaucoma surgery, conjunctival hyperemia was observed in all patients at 1-day follow-up, which all disappeared spontaneously within 1 week. One patient had scleral marks during 3-months follow-up. In addition, 2 patients presented mild corneal edema at 1-day followup, which disappeared at 7-day follow-up. No serious complications, such as hypotony, macular edema, or choroidal detachment, occurred after UCP treatment. Baseline and 6-month BCVA are shown in Table 5. BCVA at month $6(1.628 \pm 1.439 \log$ MAR $)$ was not significantly different compared with preoperative BCVA (1.634 \pm $1.446 \log \mathrm{MAR})$. There was no significant deterioration caused by UCP procedure among 17 patients with $\geq$ LP visual acuity.

\section{Discussion}

Although the treatment of glaucoma continues to update, glaucoma is still the main cause of permanent visual impairment in adults. In recent years, UCP treatment using high-intensity focused ultrasound technology 
Table 5. Patients' characteristics and results at 6 months in IOP and visual acuity

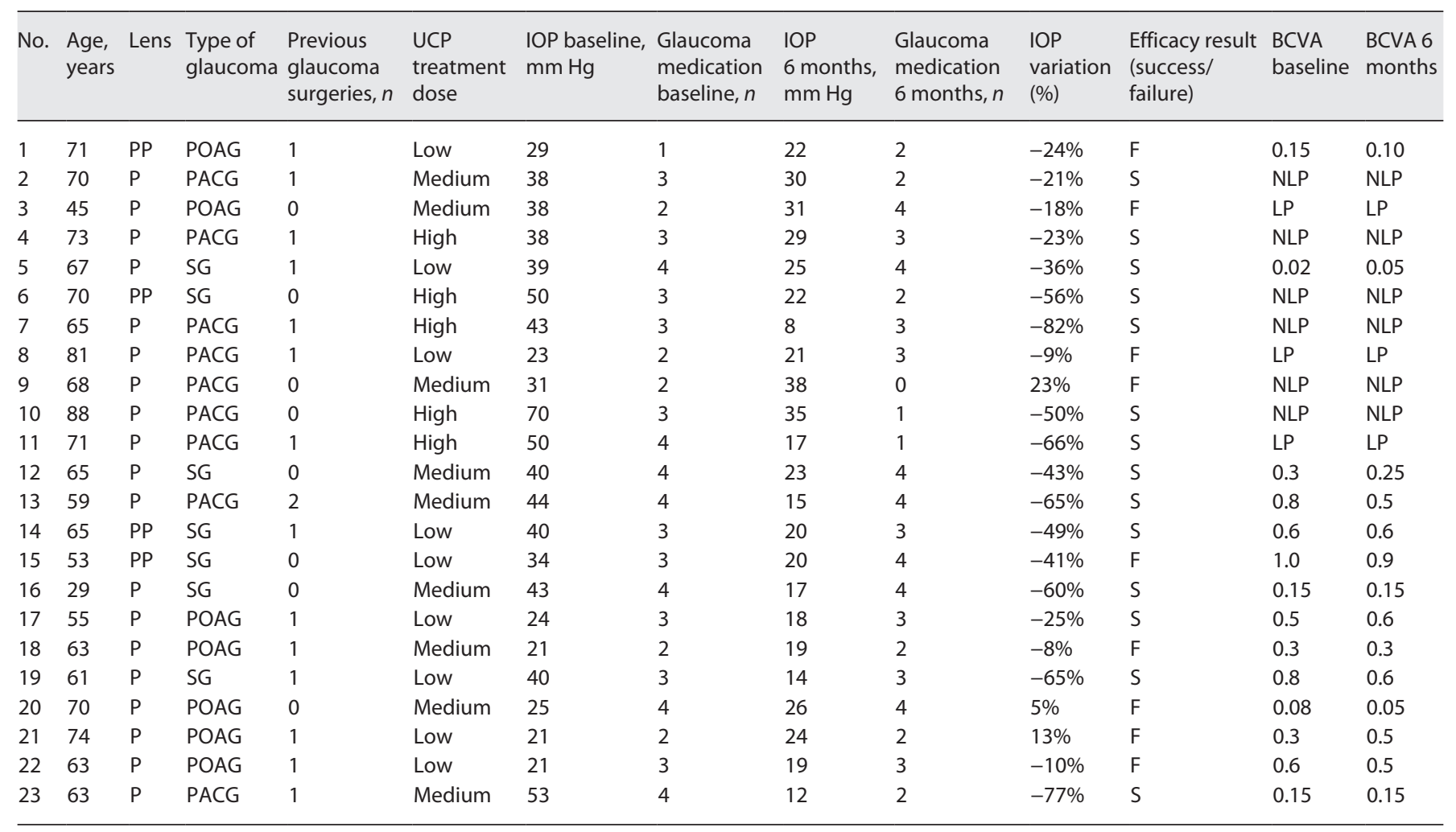

IOP, intraocular pressure; BCVA, best corrected visual acuity; NLP, no light perception; LP, light perception; P, phakic; PP, pseudophakic ; POAG, primary open-angle glaucoma; PACG, primary angle closure glaucoma; SG, secondary glaucoma; S, success; F, failure; UCP, ultrasound cycloplasty; UCP treatment dose (low $=6$ sectors; medium $=8$ sectors; and high $=10$ sectors).

has been applied to partially coagulate the ciliary body as a novel noninvasive glaucoma procedure, accompanied with a more predictable and controlled IOP reduction when compared to traditional cyclodestructive surgeries $[10,11]$. In our present study, UCP procedure achieved significant $34 \%$ IOP reduction at the 6-month follow-up visit, which was within the efficiency range from 20.1 to $40.8 \%$ published in previous studies [7]. In the previous studies in Europe, the 6-sector dose of UCP treatment was selected due to the relative lower baseline mean IOP of European glaucoma patients. According to the baseline IOP and visual acuity, there were 3 different doses of UCP procedure in our study. Patients with higher IOP were performed with 8-sector or 10-sector UCP procedure, and there was a significant positive relation between baseline IOP value and postoperative IOP reduction. As expected, patients with 10-sector UCP achieved the highest IOP reduction and therapeutic success rate, with similar safety profile and no more side effects.
In China, the incidence of PACG is the highest among glaucoma subtypes, followed by primary open-angle glaucoma and SG [2]. In a previous study of UCP in Chinese glaucoma patients [10], PACG responded much better than other types of glaucoma. Our results showed that patients with PACG and SG had achieved the greatest IOP reduction (41 and 50\%, respectively) and therapeutic success rate but will have to be confirmed by other series on a larger population. Patients with refractory glaucoma often had received glaucoma surgery, and UCP procedure has gradually become the new choice after other surgical failures. Similar to the findings of previous studies $[7,19,20]$, UCP allowed to reduced significantly the IOP in patients with or without previous glaucoma surgery (respectively from $34.9 \pm 10.9$ in baseline to $19.6 \pm 6.0$ at 6 months and from $41.4 \pm 13.8-26.5 \pm 7.5$ ), and the difference between both subgroups was not statistically significant.

Cyclodestructive methods usually induced excessive destruction by partially heating or freezing the ciliary 
body and caused additional side effects. By means of simple preoperative ocular examination and correct operation process, UCP procedure precisely positioned in the ciliary body without adjacent tissue damages. Although both UCP procedure and traditional cyclocryotherapy showed good efficacy in postoperative IOP reduction, Ruixue et al. [11] considered that UCP was safer with less postoperative complications and serious adverse effects. At present, most of the complications observed in the treatment of UCP were mild and transient, such as conjunctival hyperemia, scleral marks, corneal edema, and ocular pain [16]. As expected, our results showed that operative complications such as conjunctival hyperemia disappeared within 1 month. Moreover, as previously reported by $\mathrm{Hu}$ et al. [10], the decrease of IOP after UCP completely relieved the patients' ocular pain symptoms before operation.

Compared with other cyclodestruction procedures, UCP procedure had the highest safety features with lower incidences of mild and serious complications reported in the literature such as macular edema [7], hypotony [10], and choroidal detachment [21], and none of these had appeared in our study. There was no significant effect on patients' vision acuity. Mean visual acuity remained statistically unchanged (logMAR BCVA $1.63 \pm 1.45$ before UCP and $1.62 \pm 1.44$ at month $6[p=0.883])$. No eye demonstrated a reduction of 2 lines or more in $\mathrm{BCVA}$, and no eye underwent a reduction from LP to NLP.

Thanks to the good safety profile, multiple UCP treatment may be performed in some patients with an insufficient IOP reduction after first UCP procedure until the target IOP is reached [14, 15, 20-22]. DeGregorio et al. [15] evaluated the effects of a second and possibly a third UCP procedure in patients who responded to a first procedure but in whom the target IOP was not reached 4 months after the procedure. UCP treatments were performed only with 6 -sector protocol, and with $52.6 \%$ of IOP reduction and a complete success rate (defined by IOP $<21 \mathrm{~mm} \mathrm{Hg}$ at 1 year) achieved in 85\% (34/40 eyes treated), the authors demonstrated that multiple UCP treatments have a cumulative effect on the IOP reduction and therapeutic success rate, with a good safety profile.

Efficacy and safety of a second UCP procedure in patients who demonstrated a significant IOP decrease after the first procedure but then presented an early or a delayed increase was studied by Aptel et al. [22]. Authors concluded that a second UCP allows a sustained IOP decrease in a significant number of subjects with a safety profile comparable to the first procedure. None of pa- tients were treated a second time with UCP during the 6-month follow-up period of our study, but 7 of them had an additional procedure later.

The present study mainly includes the following limitation. First, our study is a noncomparative design. As this is a recent procedure in China and our first experience, UCP efficacy and safety profile on Chinese patients should be well established before designing and starting comparative studies with traditional filtering glaucoma surgeries such as trabeculectomy and/or other cyclodestructive procedures [11]. Second, the aim of the study was to evaluate the short-term efficacy and safety in Chinese patients, with a follow-up duration of 6 months, which can be considered relatively short, compared to published papers on UCP with 24-month follow-up on European population [7, 16]. We are continuing to follow the patients and we will report midterm and multidose results on Chinese patients later. Third, included a large population of patients with different types of glaucoma and used 3 ultrasound doses to treat patients. As UCP is a recent technology in China, we opted for a noncomparative design, but in future, we need to stratify the population to a well-established efficacy profile.

In conclusion, our study showed and confirmed that UCP procedure was an effective and well-tolerated treatment to reduce IOP in Chinese glaucoma patients. UCP offered a novel alternative for the treatment of glaucoma with better safety and easier operability and should be considered for moderate stage of glaucoma. Moreover, efficacy has been suggested to further increase with repeated procedures that should be confirmed on Chinese patients.

\section{Statement of Ethics}

All procedures performed in studies involving human participants were in accordance with the ethical standards of the institutional research committee and with the Declaration of Helsinki. Written informed consent was obtained from all individual participants included in the study. The study was approved by the Ethics Committee of the Shanghai General Hospital, Shanghai Jiao Tong University, Shanghai, China (approval number: CS18CHN-04).

\section{Conflict of Interest Statement}

The authors have no conflicts of interest to declare. 


\section{Funding Sources}

This article did not receive any funding.

\section{Author Contributions}

Q.L. and W.W. X. are joint first authors. Q.L., W.W.X., and L.G. analyzed the data and drafted the manuscript. Y.L.W. contributed to the critical revision of the manuscript. B.C., S.S.W., Y.P.D., and Y.R. collected the data. L.G. designed the study. All authors have read and approved the final manuscript.

\section{References}

1 Jonas JB, Aung T, Bourne RR, Bron AM, Ritch R, Panda-Jonas S. Glaucoma. Lancet. 2017 Nov;390(10108):2183-93.

2 Song P, Wang J, Bucan K, Theodoratou E, Rudan I, Chan KY. National and subnational prevalence and burden of glaucoma in China: a systematic analysis. J Glob Health. 2017 Dec; 7(2):020705.

3 Lusthaus J, Goldberg I. Current management of glaucoma. Med J Aust. 2019 Mar;210(4): $180-7$.

4 Rosdahl JA, Gupta D. Prospectivestudies of minimally invasive glaucoma surgeries: systematic review and quality assessment. Clin Ophthalmol. 2020 Jan;14:231-43.

5 Reznicek L, Boghos Y, Lanzl IM. [Possibilities and limitations of glaucoma surgery]. Ophthalmologe. 2016 Oct;113(10):833-7.

6 Iliev ME, Gerber S. Long-term outcome of trans-scleral diode laser cyclophotocoagulation in refractory glaucoma. Br J Ophthalmol. 2007 Dec;91(12):1631-5.

7 Posarelli C, Covello G, Bendinelli A, Fogagnolo P, Nardi M, Figus M. High-intensity focused ultrasound procedure: the rise of a new noninvasive glaucoma procedure and its possible future applications. Surv Ophthalmol. 2019 Nov-Dec;64(6):826-34.

8 Charrel T, Aptel F, Birer A, Chavrier F, Romano F, Chapelon JY, et al. Development of a miniaturized HIFU device for glaucoma treatment with conformal coagulation of the ciliary bodies. Ultrasound Med Biol. 2011 May;37(5):742-54.
9 Aptel F, Denis P, Rouland JF, Renard JP, Bron A. Multicenter clinical trial of high-intensity focused ultrasound treatment in glaucoma patients without previous filtering surgery. Acta Ophthalmol. 2016 Aug;94(5):e268-77.

$10 \mathrm{Hu}$ D, Tu S, Zuo C, Ge J. Short-term observation of ultrasonic cyclocoagulation in Chinese patients with end-stage refractory glaucoma: a retrospective study. J Ophthalmol. 2018 Sept;2018:4950318.

11 Ruixue W, Tao W, Ning L. A comparative study between ultrasound cycloplasty and cyclocryotherapy for the treatment of neovascular glaucoma. J Ophthalmol. 2020 Jan;2020: 4016536.

12 Deb-Joardar N, Reddy KP. Application of high intensity focused ultrasound for treatment of open-angle glaucoma in indian patients. Indian J Ophthalmol. 2018 Apr;66(4): $517-23$.

13 Graber M, Rothschild PR, Khoueir Z, Bluwol E, Benhatchi N, Lachkar Y. High intensity focused ultrasound cyclodestruction versus cyclodiode treatment of refractory glaucoma: A retrospective comparative study. J Fr Ophtalmol. 2018 Sept;41(7):611-8.

14 Melamed S, Goldenfeld M, Cotlear D, Skaat A, Moroz I. High-intensity focused ultrasound treatment in refractory glaucoma patients: results at 1 year of prospective clinical study. Eur J Ophthalmol. 2015 Nov-Dec: 25(6):483-9.

15 De Gregorio A, Pedrotti E, Stevan G, Montali M, Morselli S. Safety and efficacy of multiple cyclocoagulation of ciliary bodies by high-intensity focused ultrasound in patients with glaucoma. Graefes Arch Clin Exp Ophthalmol. 2017 Dec;255(12):2429-35.
16 Leshno A, Rubinstein Y, Singer R, Sher I, Rotenstreich Y, Melamed S, et al. High-intensity focused ultrasound treatment in moderate glaucoma patients: results of a 2-year prospective clinical trial. J Glaucoma. $2020 \mathrm{Jul}$; 29(7):556-60.

17 Huang XT, Qi Y, Cui QQ, Zan XN, Wang YR Zhang FY. Clinical effect and safety of ultrasound cycloplasty in the treatment of neovascular glaucoma. Int J Ophthalmol. 2020 May: 842-6.

18 Mou Dp, Tang X, An WZ, Li L, Qing GP, Shi Y. Half-year follow-up of ultrasound cycloplasty for refractory glaucoma. Chin J Ophthalmol Med. 2019 Dec;9(6):366-71.

19 Denis P, Aptel F, Rouland JF, Nordmann JP, Lachkar Y, Renard JP, et al. Cyclocoagulation of the ciliary bodies by high-intensity focused ultrasound: a 12-month multicenter study. Invest Ophthalmol Vis Sci. 2015 Jan;56(2): 1089-96.

20 Aptel F, Dupuy C, Rouland JF. Treatment of refractory open-angle glaucoma using ultrasonic circular cyclocoagulation: a prospective case series. Curr Med Res Opin. 2014 Aug; 30(8):1599-605.

21 MoraisSarmento T, Figueiredo R, Garrido J, Rebelo AL. Transient choroidal detachment after ultrasonic circular cyclocoagulation. BMJ Case Rep. 2019 Oct;12(10):e231677.

22 Aptel F, Tadjine M, Rouland JF. Efficacy and safety of repeated ultrasound cycloplasty procedures in patients with early or delayed failure after a first procedure. J Glaucoma. 2020 Jan;29(1):24-30. 